\title{
Opportunities for Asteroid Retrieval Missions
}

\author{
Pre-print proof-reading copy. The final publication is available at:
}

http://link.springer.com/chapter/10.1007/978-3-642-39244-3 21

D. García Yárnoz, J.P. Sanchez, C.R. McInnes

Advanced Space Concepts Laboratory, University of Strathclyde, UK.

\begin{abstract}
Asteroids and comets are of strategic importance for science in an effort to uncover the formation, evolution and composition of the Solar System. Near-Earth Objects (NEOs) are of particular interest because of their accessibility from Earth, but also because of their speculated wealth of material resources. The exploitation of these resources has long been discussed as a means to lower the cost of future space endeavours. In this chapter, we analyze the possibility of retrieving entire objects from accessible heliocentric orbits and moving them into the Earth's neighbourhood. The asteroid retrieval transfers are sought from the continuum of low energy transfers enabled by the dynamics of invariant manifolds; specifically, the retrieval transfers target planar, vertical Lyapunov and halo orbit families associated with the collinear equilibrium points of the Sun-Earth Circular Restricted Three Body problem. The judicious use of these dynamical features provides the best opportunity to find extremely low energy transfers for asteroidal material. With the objective to minimise transfer costs, a global search of impulsive transfers connecting the unperturbed asteroid's orbit with the stable manifold phase of the transfer is performed. A catalogue of asteroid retrieval opportunities of currently known NEOs is presented here. Despite the highly incomplete census of very small asteroids, the catalogue can already be populated with 12 different objects retrievable with less than $500 \mathrm{~m} / \mathrm{s}$ of $\Delta v$. All, but one, of these objects have an expected size in the range that can be met by current propulsion technologies. Moreover, the methodology proposed represents a robust search for future retrieval candidates that can be automatically applied to a growing survey of NEOs.
\end{abstract}




\section{Introduction}

Recently, significant interest has been devoted to the understanding of minor bodies of the Solar System, including near-Earth and main belt asteroids and comets. NASA, ESA and JAXA have conceived a series of missions to obtain data from such bodies, having in mind that their characterisation not only provides a deeper insight into the Solar System, but also represents a technological challenge for space exploration. Near Earth Objects in particular have also stepped into prominence because of two important aspects: they are among the easiest celestial bodies to reach from the Earth and they may represent a potential impact threat. This increased interest has encouraged the research community to propose further asteroid engineering projects, such as NEO retrieval missions, taking advantage of the synergies with current minor bodies search campaigns and asteroid manipulation technology development initiatives.

Various space macro-engineering projects have as a primary requirement the capture or shepherding of a portion or a full asteroid in useful orbits in the solar system (see Table 1).

Early proposals for the space elevator involved the capture of a small body in a close to GEO orbit to serve as counterweight. The size of the counterweight required depends on the radius of the orbit where the asteroid would be placed, with size decreasing exponentially with altitude above GEO, resulting in about a 50 ton asteroid (about $3.3 \mathrm{~m}$ diameter) for a counterweight at a circular orbit of radius $100000 \mathrm{~km}$.

The use of captured asteroids has also been proposed for geoengineering purposes as means of reducing the solar insolation on Earth by generating dust rings or clouds. Depending on the position of the dust cloud, either Earth ring (Pearson et al., 2006), Sun-Earth $\mathrm{L}_{1}$ (Bewick et al., 2012), or Earth-Moon $\mathrm{L}_{4} / \mathrm{L}_{5}$ region (Struck, 2007), and the desired reduction in insulation, the asteroid mass requirements and the complexity and cost of the capture transfer vary, but the minimum size for a target asteroid is never below 500 meters diameter. Retrieving objects of this size is probably beyond nowadays technological capabilities. The required mass could be reduced by more than one order of magnitude by the use of space manufactured solar reflectors instead of dust rings or clouds (Pearson et al., 2006), but that would involve a large manufacturing infrastructure on orbit.

Much smaller asteroids can already be of interest for resources exploitation. The in-situ utilisation of resources in space has long been suggested as the means of lowering the cost of space missions, by, for example, providing bulk mass for radiation shielding or distilling rocket propellant for 
interplanetary transfers (Lewis, 1996). Although the concept of asteroid mining dates back to the very first rocketry pioneers (Tsiolkovsky, 1903), evidences of a renewed interest can be found in the growing body of literature on the topic (Baoyin et al., 2010, Sanchez and McInnes, 2011a, Hasnain et al., 2012), as well as in high profile private enterprise announcements such as by Planetary Resources Inc ${ }^{1}$. A recent asteroid retrieval mission study (Brophy et al., 2012) proposed the capture of a 2-4 m diameter asteroid around the Moon with current technologies, which could serve as test-bed for the development of technologies for in-situ resource utilisation (ISRU). Other proposals (Massonnet and Meyssignac, 2006) suggest a larger asteroid to be used as a NEO shield in combination with resource exploitation. These technologies could become a potentially disruptive innovation for space exploration and utilisation and enable large-scale space ventures that could today be considered far-fetched, such as large space solar power satellites or sustaining communities in space. Fuel depots or permanent space stations that use a small asteroid as a base can be envisaged in the near future. The use of asteroids in cycler orbits to provide structural support and radiation shielding for interplanetary transfers can be considered a more futuristic enterprise also found in literature (Lewis, 1996).

Table 1: Macro-engineering projects proposing asteroid capture. To estimate asteroid sizes, given in diameter, an average NEO density of $2.6 \mathrm{gr} / \mathrm{cm}^{3}$ (Chesley et al., 2002) was used when neccesary.

\begin{tabular}{|c|c|c|c|}
\hline Project & Target orbit(s) & Size or mass required & Reference \\
\hline Space Elevator & $\sim \mathrm{GEO}$ & $52 \times 10^{3} \mathrm{~kg}(>3.3 \mathrm{~m})$ & (Aravind, 2007) \\
\hline Geo-engineering: & : LEO & $2.3 \times 10^{12} \mathrm{~kg}$ & (Pearson et al., 2006) \\
\hline Dust ring LEO & & $(>1190 \mathrm{~m})$ & \\
\hline $\begin{array}{l}\text { Geo-engineering: } \\
\text { Dust cloud } L_{1}\end{array}$ & Sun-Earth $\mathrm{L}_{1}$ & $\begin{array}{l}1.9 \times 10^{11} \mathrm{~kg} \\
(>515 \mathrm{~m})\end{array}$ & (Bewick et al., 2012) \\
\hline $\begin{array}{l}\text { Geo-engineering: } \\
\text { Dust cloud } \mathrm{L}_{4 / 5}\end{array}$ & Earth-Moon $\mathrm{L}_{4} / \mathrm{L}_{5}$ & $\begin{array}{l}2.1 \times 10^{14} \mathrm{~kg} \\
(>5.3 \mathrm{~km})\end{array}$ & (Struck, 2007) \\
\hline $\begin{array}{l}\text { Tech. demo } \\
\text { ISRU/Fuel depot }\end{array}$ & $\begin{array}{l}\mathrm{L}_{1}, \mathrm{~L}_{2}, \text { Moon or- } \\
\text { bit... }\end{array}$ & $>2 \mathrm{~m}$ & (Brophy et al., 2012) \\
\hline Space station & $\mathrm{L}_{1}, \mathrm{~L}_{2}, \mathrm{~L}_{4}, \mathrm{~L}_{5}$ & $>10 \mathrm{~m}$ & \\
\hline NEO shield & Sun-Earth $\mathrm{L}_{1}, \mathrm{~L}_{2}$ & $20-40 \mathrm{~m}$ & $\begin{array}{l}\text { (Massonnet and } \\
\text { Meyssignac, 2006) }\end{array}$ \\
\hline Cyclers & $\begin{array}{l}\text { Earth-Mars reso- } \\
\text { nant orbit }\end{array}$ & $>100 \mathrm{~m}$ & (Lewis, 1996) \\
\hline
\end{tabular}

\footnotetext{
${ }^{1}$ http://www.planetaryresources.com/
} 
In all these studies, the Sun-Earth Lagrangian points repeatedly appear as one of the preferred destinations for captured asteroids. This is relevant as they can also serve as natural gateways to other destinations in the Earth-Moon system through the use of heteroclinic connections (Koon et al., 2000). There are however several main challenges to overcome: the development of techniques to modify asteroid trajectories, the improvement in the minor body census in order to find the most suitable candidates of the appropriate size, and the design of low-cost transfers to the desired final orbits.

Current technologies and methods for deflection of Earth-impacting objects have experienced significant advances, along with increasing knowledge of the asteroid population. While initially devised to mitigate the hazard posed by global impact threats, the current impact risk is largely posed by the population of small undiscovered objects (Shapiro et al., 2010), and thus methods have been discussed to provide subtle orbital changes to these small objects, as opposed to large-scale interventions, e.g., the use of nuclear devices (Kleiman, 1968). This latter batch of deflection methods, such as low thrust tugboat (Scheeres and Schweickart, 2004), gravity tractor (Edward and Stanley, 2005) or small kinetic impactor (Sanchez and Colombo, 2012) are moreover based on currently proven space technologies. They may therefore render the apparently ambitious scenario of manipulating asteroid trajectories a likely option for the near future.

With regards the accessibility of asteroid resources, recent work by Sanchez and McInnes (2011a, 2012) demonstrates that a substantial quantity of resources can indeed be accessed at relatively low energy; on the order of $10^{14} \mathrm{~kg}$ of material could potentially be harvested at an energy cost lower than that required to access the resources of the Moon. In their work, the accessibility of asteroid material is estimated by analysing the volume of Keplerian orbital element space from which the Earth can be reached under a given energy threshold by means of a bi-impulsive transfer. This volume of Keplerian element space is then mapped into existing NEO orbital (Bottke et al., 2002) and size distribution models (Mainzer et al., 2011). As discussed in Sanchez and McInnes (2011a, 2012), it is perhaps more important that asteroid resources can be accessed across a wide spectrum of energies, and thus, as shown in Sanchez and McInnes (2012), current technologies could be adapted to return to the Earth's neighbourhood objects from 10 to 30 meters diameter for scientific exploration and resource utilisation purposes.

Advances in both asteroid deflection technologies and dynamical system theory, which allow new and cheaper means of space transportation, are now enabling radically new mission concepts, including but not limited to asteroid retrieval missions. These envisage a spacecraft reaching a suitable 
object, attaching itself to the surface and returning it, or a portion of it, to the Earth's orbital neighbourhood. Moving an entire asteroid into an orbit in the vicinity of Earth entails an obvious engineering challenge, but may also allow a much more flexible exploitation phase in the Earth's neighbourhood.

The work presented here aims to provide a feasibility assessment of the latter mission concept by defining a set of preliminary mission opportunities that could be enabled by invariant manifold dynamics. Missions delivering a large quantity of material to the Lagrangian points are of particular interest. The material can be used in a first stage as test bed for ISRU technology demonstration missions and material processing at affordable costs. The science return is also greatly improved, with an asteroid permanently, or for a long duration, available for study and accessible to telescopes, probes and even crewed missions to the Lagrangian points. Finally, it sets the stage for other future endeavours, such as the ones listed in Table 1.

\section{Low Energy Transport Conduits}

Current interplanetary spacecraft have masses on the order of $10^{3} \mathrm{~kg}$, while a small body of just 10 meters diameter will most likely have a mass of the order of $10^{6} \mathrm{~kg}$. Hence, already moving such a small object, or an even larger one, with the same ease that a scientific payload is transported today, would demand propulsion systems orders of magnitudes more powerful and efficient; or alternatively, orbital transfers orders of magnitude less demanding than those to reach other planets in the solar system.

Solar system transport phenomena, such as the rapid orbital transitions experienced by comets Oterma and Gehrels 3, from heliocentric orbits with periapsis outside Jupiter's orbit to apoapsis within Jupiter's orbit, or the Kirkwood gaps in the main asteroid belt, are some manifestations of the sensitivities of multi-body dynamics. The same underlying principles that enable these phenomena allow also excellent opportunities to design surprisingly low energy transfers.

It has for some time been known that the hyperbolic invariant manifold structures associated with periodic orbits around the $\mathrm{L}_{1}$ and $\mathrm{L}_{2}$ collinear points of the Three Body Problem provide a general mechanism that controls the aforementioned solar system transport phenomena (Koon et al., 2000). In this analysis, we seek to benefit from these mathematical constructs in order to find low-cost trajectories to retrieve asteroid material to the Earth's vicinity. This work assumes the motion of the spacecraft and asteroid under the gravitational influence of Sun and Earth, within the 
framework of the Circular Restricted Three Body Problem (CR3BP) (Koon et al., 2008). The well known equilibrium points of the system are shown in Fig. 1. The mass parameter $\mu$ considered in this analysis is $3.0032080443 \times 10^{-6}$, which neglects the mass of the Moon. Note that the usual normalised units are used when citing Jacobi constant values (Koon et al., 2008).

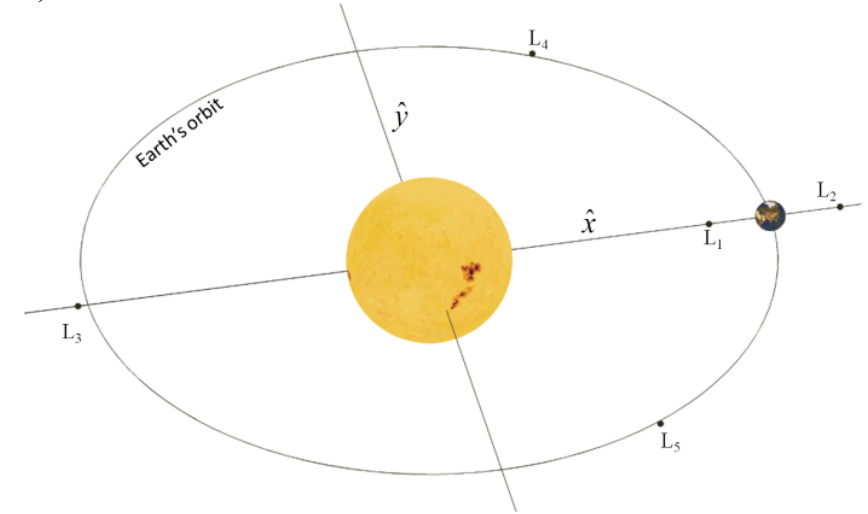

Fig. 1: Schematic of the CR3BP and its equilibrium points.

\section{Periodic Orbits and Manifold Structure}

In particular, we are interested in the dynamics concerning the Sun-Earth $\mathrm{L}_{1}$ and $\mathrm{L}_{2}$ points (see Fig. 1), as they are the gate keepers for potential ballistic capture of asteroids in the Earth's vicinity.

During the last half a century there has been an intense effort to catalogue all bounded motion near the libration points of the Circular Restricted Three Body Problem (Howell, 2001). The principal families of bounded motion that have been discovered are planar and vertical Lyapunov periodic orbits, quasi-periodic Lissajous orbits, and periodic and quasi-periodic halo orbits (Gómez et al., 2000, Koon et al., 2008). Some other families of periodic orbits can be found by exploring bifurcations in the aforementioned main families (Howell, 2001).

Theoretically, an asteroid transported into one of these orbits would remain near the libration point for an indefinite time. In practice, however, these orbits are unstable, and an infinitesimal deviation from the periodic orbit will make the asteroid depart asymptotically from the libration point regions. Nevertheless, small correction manoeuvres can be assumed to be able to keep the asteroid within the periodic orbit (Simó et al., 1987, Howell and Pernicka, 1993). 
The linear behaviour of the motion near the libration points is of the type centre x centre x saddle (Szebehely, 1967). All bounded motion near these points arises from the stable centre $\mathrm{x}$ centre behaviour, while the saddle dynamical behaviour ensures that, inherent to any bounded trajectory near the libration points, an infinite number of trajectories exist that asymptotically approach, or depart from, the bounded motion. All these sets of trajectories, both bounded and unbounded motion, associated to a libration point form what is called the invariant manifold structure (Gómez et al., 2005).

There are two classes of invariant manifolds: the central invariant and the hyperbolic invariant. The central invariant manifold is composed of periodic and quasi-periodic orbits near the libration points, while the hyperbolic invariant manifold consists of a stable and an unstable set of trajectories associated with an unstable orbit near an equilibrium point. The unstable manifold is formed by the infinite set of trajectories that exponentially leaves the periodic or quasi-periodic motion to which they are associated. The stable manifold, on the other hand, consists of an infinite number of trajectories exponentially approaching the periodic or quasi-periodic orbit.

It is well known (e.g., Koon et al. (2008)) that the phase space near the equilibrium regions can be divided into four broad classes of motion; bound motion near the equilibrium position (i.e., periodic and quasiperiodic orbits), asymptotic trajectories that approach or depart from the latter, transit trajectories, and, non-transit trajectories (see Fig. 2). A transit orbit is a trajectory such that its motion undergoes a rapid transition between orbiting regions. In the Sun-Earth case depicted in Fig. 2, for example, the transit trajectory approaches Earth following a heliocentric trajectory, transits through the bottle neck delimited by the halo orbit and becomes temporarily captured at Earth. An important observation from dynamical system theory is that the hyperbolic invariant manifold structure defined by the set of asymptotic trajectories forms a phase space separatrix between transit and non-transit orbits.

It follows from the four categories of motion near the libration points that periodic orbits near the Sun-Earth $\mathrm{L}_{1}$ and $\mathrm{L}_{2}$ points can not only be targeted as the final destination of asteroid retrieval missions, but also as natural gateways of low energy trajectories to Earth centred temporarily captured trajectories or transfers to other locations of the cis-lunar space, such as the Earth Moon Lagrangian points.

This work will focus in capture opportunities to periodic orbits near the libration points, which will be enabled by the hyperbolic stable manifold trajectories associated with them. In particular, we will focus on three distinct classes of periodic motion near the Sun-Earth $\mathrm{L}_{1}$ and $\mathrm{L}_{2}$ points; Planar 
and Vertical Lyapunov and Halo Orbits, from now on referred to as a whole as libration point orbits (LPO). This analysis seeks a first insight into the future feasibility of asteroid retrieval missions, and thus we will limit our search to these three classic families of bounded motion near the Sun-Earth $\mathrm{L}_{1}$ and $\mathrm{L}_{2}$ points. Future and more comprehensive searches however should extend to capture trajectories to quasi-periodic orbits, such as Lissajous and quasi-halos. These more exotic families of eventually periodic orbits and their associated sets of asymptotic trajectories will likely increase the range of options for gravitational capture of asteroids.

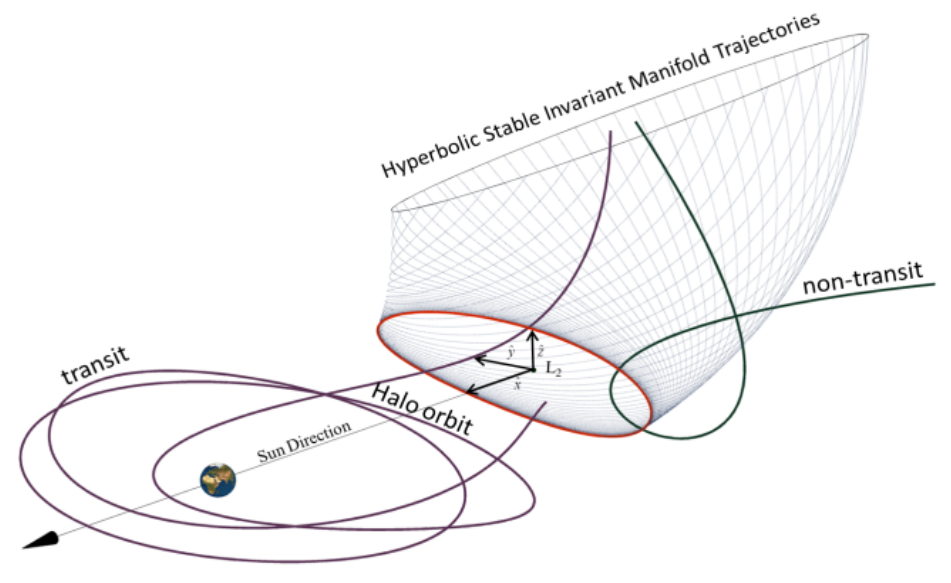

Fig. 2: Schematic representation of the four categories of motion near the $\mathrm{L}_{2}$ point (represented by the set of axes in the figure): periodic motion around $\mathrm{L}_{2}$ (i.e., halo orbit), hyperbolic invariant manifold structure (i.e., set of stable hyperbolic invariant manifold trajectories), transit trajectory and non transit trajectory.

\section{Lyapunov Orbits}

As noted earlier, the linear behaviour of the motion near the $\mathrm{L}_{1}$ and $\mathrm{L}_{2}$ points is of the type centre $\mathrm{x}$ centre $\mathrm{x}$ saddle. The centre $\mathrm{x}$ centre part generates a 4-dimensional central invariant manifold around each collinear equilibrium point when all energy levels are considered (Gómez et al., 2005). In a given energy level the central invariant manifold is a 3dimensional set of periodic and quasi-periodic solutions lying on an invariant torus, together with some chaotic or stochastic regions in between (Gómez et al., 2001). There exist families of periodic orbits with frequencies related to both centers: $\omega_{p}$ and $\omega_{v}$ (Alessi, 2010). They are known as planar Lyapunov family and vertical Lyapunov family, see Fig. 3 , and their existence is ensured by the Lyapunov center theorem. Halo 
orbits are 3-dimensional periodic orbits that emerge from the first bifurcation of the planar Lyapunov family.

To generate the entire family of planar and vertical Lyapunov periodic orbits, we start by generating an approximate solution with the associated frequency very close to the libration point (Howell, 2001). This initial solution is corrected in the non-linear dynamics by means of a differential correction algorithm (Koon et al., 2008) over a suitable plane section that takes advantage of the known symmetries of these orbits (Zagouras and Markellos, 1977). Once one periodic solution has been computed, the complete family can be generated by means of numerical continuation process that uses the previous solution as initial guess for a periodic orbit on which one of the dimension on the phase space has been perturbed slightly. By properly choosing the phase space direction that we want to continuate; and by repeating the process iteratively one can build a family of periodic orbits with increasing Jacobi constant, as shown in Fig. 3.

\section{Halo Orbits}

The term halo orbit refers to the orbit's ring shape and its position relative to the secondary mass, which reminds of the ring of light commonly used in religious iconography to denote holiness. The term was coined by Robert Farquhar, who advocated the use of these orbits near the EarthMoon $\mathrm{L}_{2}$ point to obtain a continuous communication relay with the far side of the Moon during the Apollo programme (Farquhar, 1967).
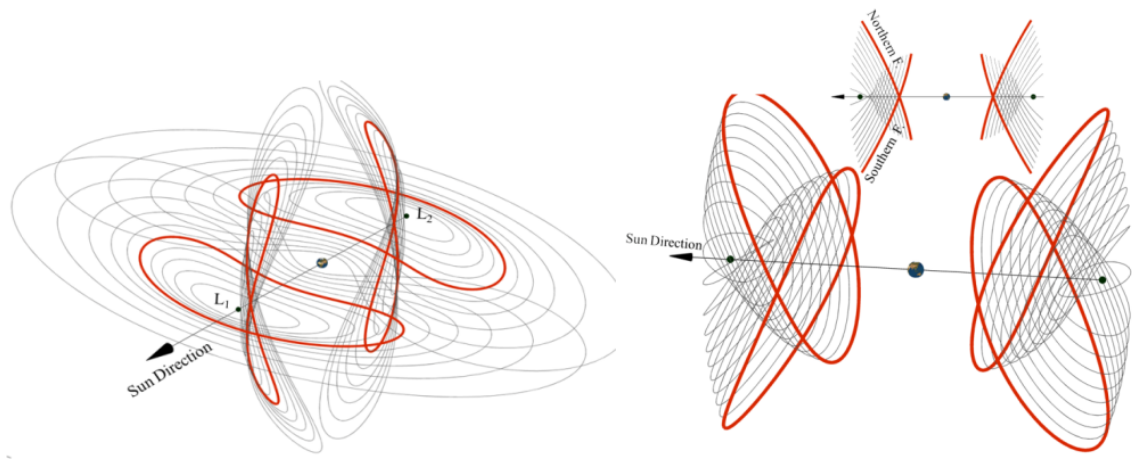

Fig. 3: Series of Planar and Vertical Lyapunov orbits (left) and northern and southern halo orbits (right) associated with the Sun-Earth $\mathrm{L}_{1}$ and $\mathrm{L}_{2}$ points. Lyapunov orbits are plotted ranging from Jacobi constant 3.0007982727 to 3.0000030032. Halo orbits are plotted ranging from Jacobi constant of 3.0008189806 to 3.0004448196 . The thicker red line corresponds to a Jacobi constant of 3.0004448196, which corresponds to half the distance between the energy at equilibrium in $\mathrm{L}_{2}$ and $\mathrm{L}_{3}$. 
As previously noted, this type of orbit emerges from a bifurcation in the planar Lyapunov orbits. As the amplitude of planar Lyapunov orbit increases, eventually a critical amplitude is reached where the planar orbits become vertical critical, as defined by Hénon (1973), and new threedimensional families of periodic orbits bifurcate. Thus, the minimum possible size for halo orbits in the Sun-Earth system is approximately $(240 \mathrm{x}$ $660) \cdot 10^{3} \mathrm{~km}$ at $\mathrm{L}_{1}$ and $(250 \times 675) \cdot 10^{3} \mathrm{~km}$ at $\mathrm{L}_{2}$, sizes denoting the maximum excursion from the libration point in the $x$ and $y$ directions respectively. At the bifurcation point, two symmetric families of halo orbits emerge at each libration point, here referred to as the northern and southern family depending on whether the maximum $z$ displacement is achieved in the northern (i.e., $z>0$ ) or southern (i.e., $z<0$ ) direction, respectively (see Fig. 3).

Similarly to planar and vertical Lyapunov, the set of halo orbits, also shown in Fig. 3, was computed by means of the continuation of a predictor-corrector process. The initial seed was computed by means of Richardson (1980) third order approximation of a halo orbit. A differential corrector procedure is used to trim Richardson's prediction and obtain the smallest halo possible (Zagouras and Markellos, 1977, Koon et al., 2008). We then continue the process by feeding the next iteration with a prediction of a slightly larger displacement in $z$. Iteratively repeating this process provides a series of halo orbits with increasing energy, or decreasing Jacobi constant.

The process can only be continued until a Jacobi constant not far below 3.0004. At this point the direction of the continuation should be changed to the $x$ direction, or a more sophisticated processes of continuation on which the direction is modified at each iteration should be used (Ceriotti and McInnes, 2012). For this analysis however we chose to stay on the range of halo orbits that can be continued using only the $z$ direction to ensure that each halo orbit is defined by a single Jacobi constant. If halo families are continued beyond that point, they become degenerate in energy since a particular Jacobi constant defines more than one halo orbit.

\section{Asteroid Retrieval Missions}

In the past few years, several space missions have already attempted to return samples from the asteroid population (e.g., Hayabusa (Kawaguchi et al., 2008)) and others are planned for the near future ${ }^{2}$. As shown by Sanchez and McInnes (2011a, 2012), given the low transport cost expected for

${ }^{2}$ http://www.nasa.gov/topics/solarsystem/features/osiris-rex.html (last accessed 02/05/12) 
the most accessible objects, it is also possible to envisage the possibility to return to Earth entire small objects with current or near-term technology. The main challenge resides on the difficulties inherent in the detection of these small objects. Thus, for example, only 1 out of every million objects with diameter between 5 to 10 meters is currently known and this ratio is unlikely to change significantly in the coming years (Veres et al., 2009).

In this section then, we will focus our attention to the surveyed population of asteroids in search of the most accessible candidates for near-term asteroid retrieval missions by means of invariant hyperbolic stable manifolds trajectories.

For this purpose, a systematic search of capture candidates among catalogued NEOs was carried out, selecting the $\mathrm{L}_{1}$ and $\mathrm{L}_{2}$ regions as the target destination for the captured material. This gives a grasp and better understanding of the possibilities of capturing entire NEOs or portions of them in a useful orbit, and demonstrates a method that can be applied to newly discovered small bodies in the future when detection technologies improve.

\section{Invariant Manifold Trajectories to $L_{1}$ and $L_{2}$}

The design of the transfer from the asteroid orbit to the $\mathrm{L}_{1}$ and $\mathrm{L}_{2} \mathrm{LPO}$ consists of a ballistic arc, with two impulsive burns at the start and end, intersecting a hyperbolic stable invariant manifold asymptotically approaching the desired periodic orbits. These results consider only the inbound leg of a full capture mission.

Planar Lyapunov, vertical Lyapunov, and halo orbits around $\mathrm{L}_{1}$ and $\mathrm{L}_{2}$ generated with the methods described in previous sections were considered as target orbits. The invariant stable manifold trajectories leading to each of these LPO, computed by perturbing the periodic solutions on the stable eigenvector direction (Koon et al., 2008) by a magnitude of $10^{-6}$, in normalized units, were propagated backwards in the Circular Restricted 3Body Problem until they reached a fixed section in the Sun-Earth rotating frame. We refer as the invariant manifold transfer time as this propagation time. The section was arbitrarily selected as the one forming an angle of $\pm \pi / 8$ with the Sun-Earth line ( $\pi / 8$ for the $\mathrm{L}_{2}$ orbits, see Fig. 4 , the symmetrical section at $-\pi / 8$ for those targeting $\mathrm{L}_{1}$ ). This corresponds roughly to a distance to Earth of the order of $0.4 \mathrm{AU}$, where the gravitational influence of the planet is considered small. No additional perturbations were considered in the backward propagation.

In this analysis, Earth is assumed to be in a circular orbit 1 AU away from the Sun. This simplification allows the orbital elements of the mani- 
fold trajectories (and in particular at the selected section) to be independent of the insertion time into the final orbit. The only exception is the longitude of the perihelion, i.e., the sum of the right ascension of the ascending node and the argument of perihelion, which varies with the insertion time with respect to a reference time with the following relation:

$$
(\Omega+\omega)=\left(\Omega_{R E F}+\omega_{R E F}\right)+\frac{2 \pi}{T}\left(t-t_{R E F}\right)
$$

where $\Omega_{R E F}$ and $\omega_{R E F}$ are the right ascension of the ascending node and the argument of perihelion at the $\pm \pi / 8$ section for an insertion into a target orbit at reference time $t_{R E F}$, and $T$ is the period of the Earth. This variation along the Earth orbit has direct implications in the phasing costs and influences the optimal point for final insertion.

For orbits with non-zero inclination, the argument of perihelion of the manifolds is also independent of the insertion time and the above equation indicates a variation in $\Omega$. However, in the case of planar Lyapunov with zero inclination, $\Omega$ is not defined and an arbitrary value of zero can be selected, resulting in the equation representing a change in argument of perihelion.

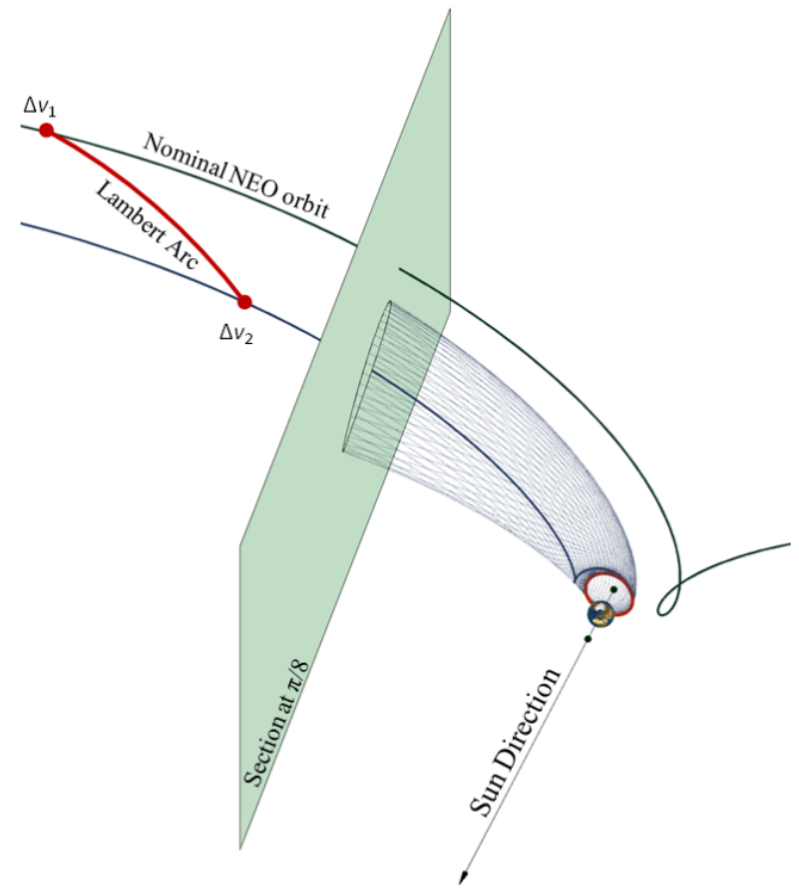

Fig. 4: Schematic representation of a transfer to $L_{2}$ 


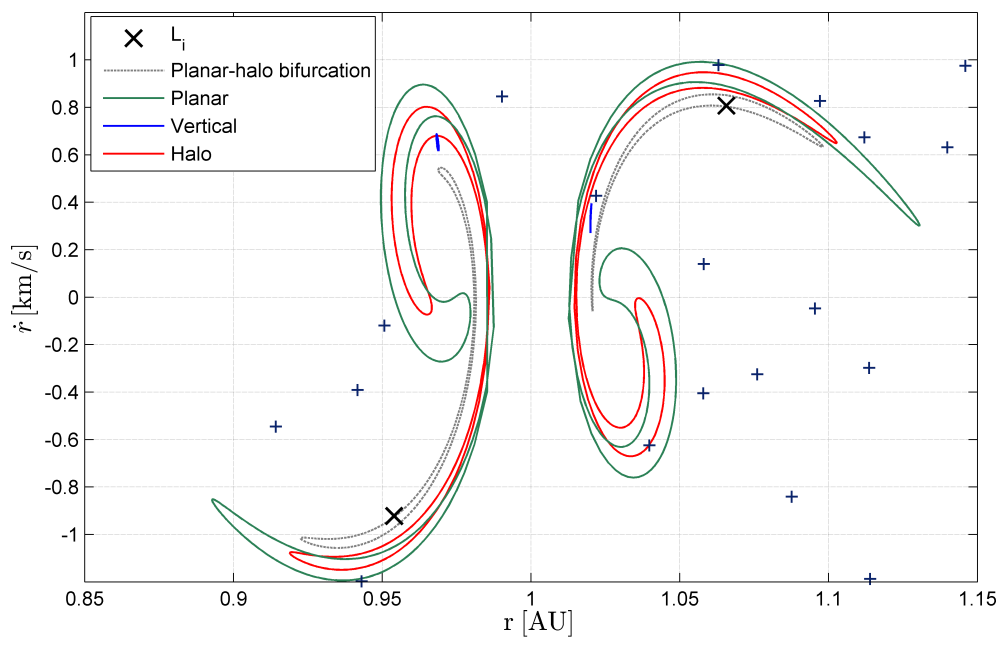

Fig. 5: Shape of the manifolds in the $r-\dot{r}$ phase space for a Jacobi constant of 3.0004448196. The manifolds are represented at their intersection with a plane forming a $\pm \pi / 8$ angle with the Sun-Earth line in the rotating frame. Manifolds on the left correspond to $\mathrm{L}_{1}$, on the right to $\mathrm{L}_{2}$. Candidate NEOs are indicated with a + marker.

The transfer between the NEO orbit and the manifold is then calculated as a heliocentric Lambert arc of a restricted two body problem with two impulsive burns, one to depart from the NEO, the final one for insertion into the manifold, with the insertion constrained to take place before or at the $\pm \pi / 8$ section.

The benefit of such an approach is that the asteroid is asymptotically captured into a bound orbit around a collinear Lagrangian point, with no need for a final insertion burn at arrival. All burns are performed far from Earth, so no large gravity losses need to be taken into account. Furthermore, this provides additional time for corrections, as the dynamics in the manifold are "slow" when compared to a traditional hyperbolic approach. Finally, this type of trajectory is then easily extendable to a low-thrust trajectory if the burns required are small.

The shape of the manifolds in the $r-\dot{r}$ phase space (with $\mathrm{r}$ being the radial distance from the Sun) at the intersection with the $\pm \pi / 8$ section is shown in Fig. 5 for a particular Jacobi constant. For an orbit with exactly the energy of $\mathrm{L}_{1}$ or $\mathrm{L}_{2}$, the intersection is a single point; while for lower Jacobi constants, the shape of the intersection is a closed loop. The intersection corresponding to the bifurcation between planar and halo orbits is also plotted. A few capture candidate asteroids have been included in the plot ( + markers) at their intersection with the $\pi / 8$ plane near their next 
closest approach to Earth. In a planar case, this would already provide a good measure of the distance of the asteroid to the manifolds. However, as we are considering the $3 \mathrm{D}$ problem, information on the $\mathrm{z}$ component or the inclination would also be necessary.
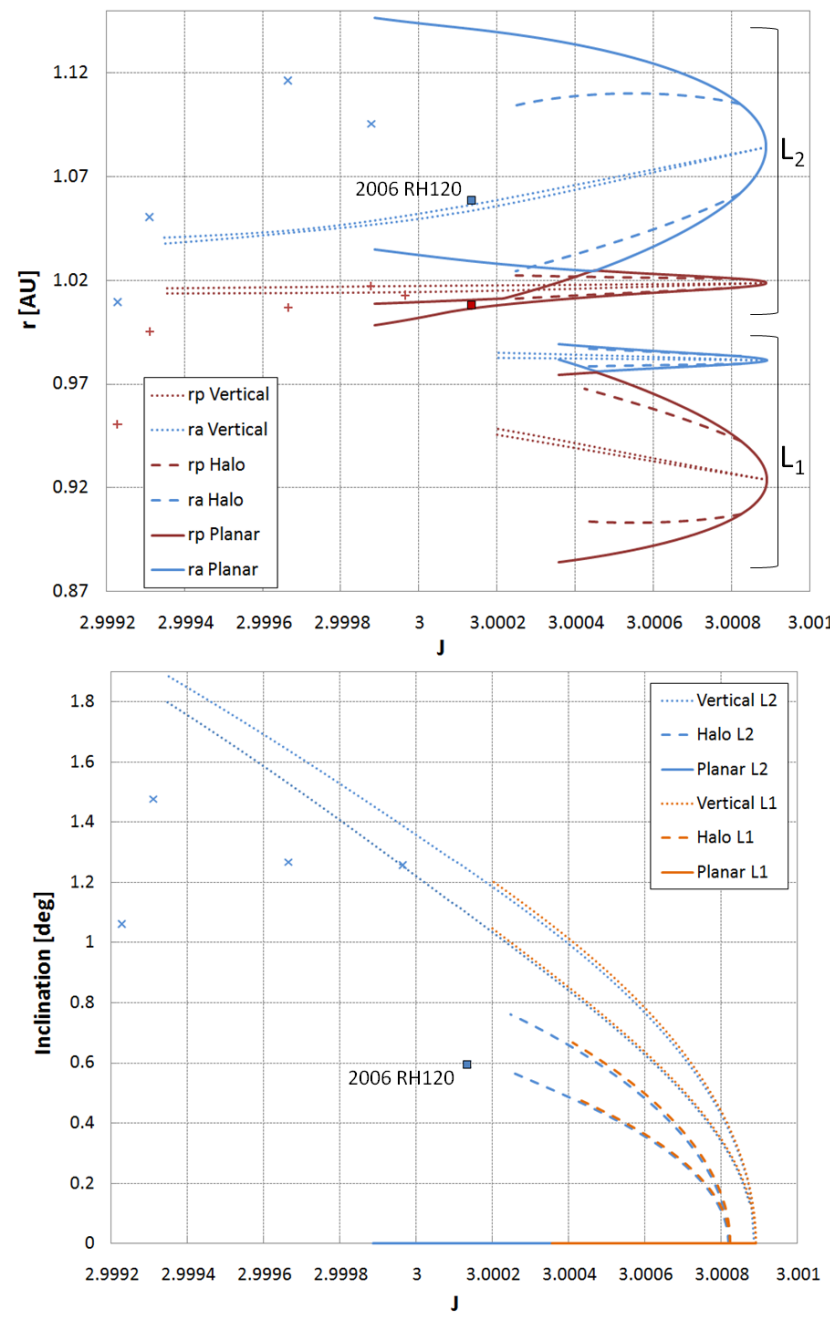

Fig. 6: Minimum and maximum perihelion and aphelion radius (top) and inclination (bottom) of the manifolds leading to planar Lyapunov, vertical Lyapunov and halo orbits around $\mathrm{L}_{1}$ and $\mathrm{L}_{2}$.

Fig. 6 provides a more useful representation of the manifolds in terms of perihelion, aphelion radius and inclination for the two collinear points. The point of bifurcation between the planar Lyapunov and halo orbits, when 
they start growing in inclination, can easily be identified. Halo orbits extend a smaller range in aphelion and perihelion radius when compared to planar Lyapunov. Vertical Lyapunov orbits have even smaller excursions in radius from a central point, as can already be seen in the smaller loops of vertical Lyapunov in Fig. 5, but on the other hand they extend to much lower values of the Jacobi constant and cover a wider range of inclinations.

Several asteroids are also plotted with small markers in the graphs. Their Jacobi constant $J$ is approximated by the Tisserand parameter as defined in Eq. (2).

$$
J \approx \frac{1}{a}+2 \sqrt{a\left(1-e^{2}\right) \cos i}
$$

where $a, e$ and $i$ are the semi-major axis (in AU), eccentricity and inclination of the asteroid orbit.

This illustrates the proximity to the manifolds of a number of NEOs. In particular, asteroid $2006 \mathrm{RH} 120$ has been highlighted, due to its proximity to the $\mathrm{L}_{2}$ manifolds. From these graphs and ignoring any phasing issues, it can already be identified as a good capture candidate, as its perihelion and aphelion radius is close to or within the range of all the three types of manifolds, and its inclination lies also close to the halo orbit manifolds. The manifold orbital elements appear to be a good filter to prune the list of NEOs to be captured.

\section{Asteroid Catalogue Pruning}

For the calculation of capture opportunities, the NEO sample used for the analysis is JPL's Small Bodies Database future ${ }^{3}$, downloaded as of $27^{\text {th }}$ of July of 2012, containing 9142 small bodies. This database represents the catalogued NEOs up to that date, and as such it is a biased population, most importantly in size, as already noted. A large number of asteroids of the most ideal size for capture have not yet been detected, as current detection methods favour larger asteroids. Secondly, there is an additional detection bias related to the type of orbits, with preference for Amors and Apollos in detriment to Atens or Atiras, as object in Aten/Atira orbits spend more time in the exclusion zone due to the Sun.

Even with this reduced list, it is a computationally expensive problem and preliminary pruning becomes necessary. Previous work by Sanchez et al. (2012) showed that the number of known asteroids that could be cap-

\footnotetext{
${ }^{3}$ http://ssd.jpl.nasa.gov/sbdb.cgi (last accessed 27/07/12)
} 
tured from a hyperbolic approach with a total $\Delta v$ less than $400 \mathrm{~m} / \mathrm{s}$ is of the order of 10. Although their hyperbolic capture approach, which roughly estimates $\Delta v$-cost for capture as the $\Delta v$ necessary to reduce the asteroid two-body energy to zero, ensuring a temporal capture, is inherently different than the manifold capture presented in this work, the number of bodies that could be captured in manifold orbits at low cost is expected to be of the same order.

Without loss of generality, it is possible to immediately discard NEOs with semi-major axis (and thus energy) far from the Earth's, as well as NEOs in highly inclined orbits. However, a more systematic filter needed to be devised.

As a first approximation of the expected total cost in terms of $\Delta v$, a biimpulsive cost prediction with both burns assumed at aphelion and perihelion was implemented. Either of the two burns is also responsible for correcting the inclination. The $\Delta v$ required to modify the semi-major axis can be expressed as:

$$
\Delta v_{a}=\sqrt{\mu_{s}\left(\frac{2}{r}-\frac{1}{a_{f}}\right)}-\sqrt{\mu_{s}\left(\frac{2}{r}-\frac{1}{a_{0}}\right)}
$$

where $\mu_{S}$ is the Sun's gravitational constant, $a_{0}$ and $a_{f}$ are the initial and final semimajor axis before and after the burn, and $r$ is the distance to the Sun at which the burn is made (perihelion or aphelion distance). On the other hand the $\Delta v$ required to modify the inclination is given by:

$$
\Delta v_{i}=2 \sqrt{\frac{\mu_{s}}{a_{0}} r^{*}} \sin (\Delta i / 2)
$$

where $\Delta i$ is the required inclination change, and $r^{*}$ corresponds to the ratio of perihelion and aphelion distance if the burn is performed at aphelion, or its inverse if performed at perihelion. These formulas only take into consideration the shape and inclination of the orbits, ignoring the rest of the orbital elements: right ascension of the ascending node and argument of pericentre. It is then implicitly assumed that the line of nodes coincides with the line of apsis and the inclination change can be performed at pericentre or apocentre. This can result in an underestimation of the plane change for some cases.

The total cost is then calculated as:

$$
\Delta v_{t}=\sqrt{\Delta v_{a 1}^{2}+\Delta v_{i 1}^{2}}+\sqrt{\Delta v_{a 2}^{2}+\Delta v_{i 2}^{2}}
$$


with one burn performed at each of the apsis, and one of the two inclination change $\Delta v$ assumed zero.

The estimated transfer $\Delta v$ corresponds thus to the minimum of four cases: aphelion burn modifying perihelion and inclination followed by a perihelion burn modifying aphelion, perihelion burn modifying aphelion and inclination followed by an aphelion burn modifying perihelion, and the equivalent ones in which the inclination change is done in the second burn.

It is important to note that these formulas are only first order approximations intended for the pruning of the database, and they will not be used to calculate the final transfers. In particular, the plane change is only valid for small changes in inclination and large deviations from the values provided by the filter are expected to be observed for high inclinations. Nevertheless, we are interested in low cost transfers which imply a small plane change, so the approximation is acceptable for filtering purposes.

For simplicity, the target manifold final perihelion, aphelion and inclination values are selected as ranges or bands obtained from Fig. 6. For example, planar Lyapunov orbits at $\mathrm{L}_{2}$ have range of $\left\{r_{p}, r_{a}, i\right\} \in\{1.00$ $1.02,1.02-1.15,0\}$, or $\{1.01-1.02,1.025-1.11,059-0.78\}$ for halo orbits at $\mathrm{L}_{2}$. Note that the inclination range for halos was given as the range corresponding to the highest energy. This is due to the fact that most candidate asteroids have higher energies that the manifolds, and the lowest cost is assumed to take place where the energy difference is minimum. In the case of vertical Lyapunov orbits, due to the narrow ranges and strong dependency with $J$, polynomial fits for $\left\{r_{p}, r_{a}, i\right\}$ as a function of $J$ were used.

With this filter, it is then possible to calculate the regions of a threedimensional orbital element space (in semi-major axis, eccentricity and inclination) than can be captured under a certain $\Delta v$ threshold. These regions are plotted in Fig. 7 for transfers to LPOs around $\mathrm{L}_{2}$ with a $\Delta v$ of $500 \mathrm{~m} / \mathrm{s}$, and any asteroid with orbital elements inside them could in principle be captured at that cost or lower. The figure shows a threedimensional view of the surfaces that delimit the regions for planar Lyapunov, vertical Lyapunov and Halo, as well as two-dimensional projections in the $a-i$ and $e-i$ planes. There is a significant overlap between the regions of different LPO target orbits; therefore, it is expected that several asteroids would allow low-cost captures to more than one family of LPO. A similar plot can be generated for the case of $\mathrm{L}_{1}$. Figure 8 presents the regions for $\mathrm{L}_{1}$ and $\mathrm{L}_{2}$ compared to the definitions of the 4 families of NEOs. Objects from all four families seem to be adequate candidates for asteroid retrieval missions, particularly the ones closed to the ApolloAmor and Aten-Atira divides. 

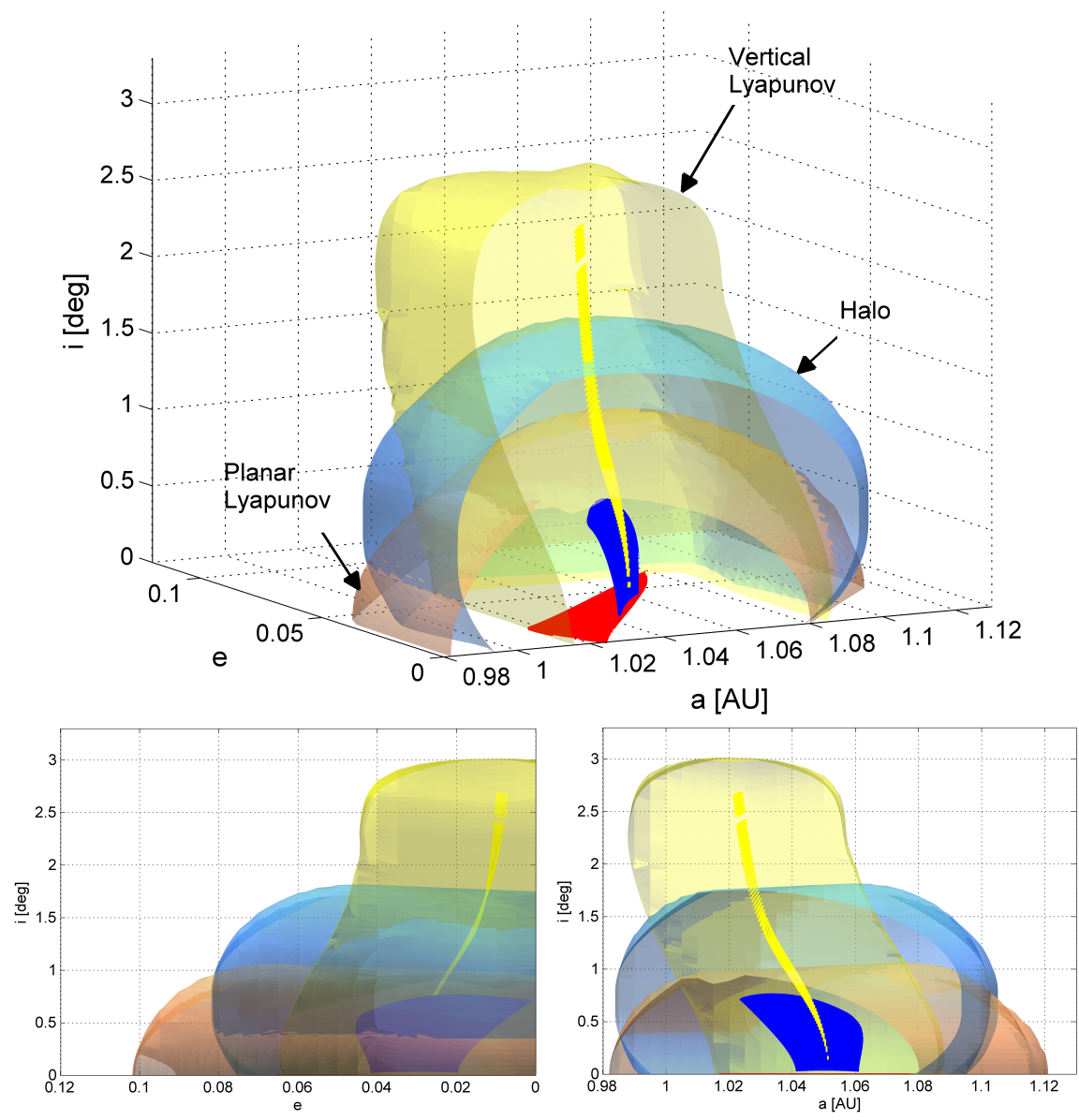

Fig. 7: Regions in the orbital element space with total estimated cost for capture into an LPO around $\mathrm{L}_{2}$ below $500 \mathrm{~m} / \mathrm{s}$. The manifolds corresponding to the LPOs are plotted in solid colours.

The filter approximation provides in general a lower bound $\Delta v$ estimate, as it ignores any phasing issues, and assumes the burns can be performed at apocentre or pericentre. Moreover, there is no guarantee, and in fact it is quite unlikely, that a combination of the extremes of the ranges of $\left\{r_{p}, r_{a}, i\right\}$ used in the filter correspond to proper manifold trajectories. Finally, the plane change does not include a modification in right ascension of the ascending node. Although the final $\Omega$ can be tuned by modifying the phasing with the Earth, this is not completely free as the final insertion will take place around a natural close approach of the asteroid with the planet. The combination of this constrained phasing and the plane change will also 
incur in additional costs. North and south halo obits provide two opportunities with opposite $\Omega$ for each transfer, which should result in two different costs, while the filter provides a single value.

For a few cases, with high initial inclination and associated plane change cost, the filter can over-estimate the $\Delta v$. As the inclination increases, solutions splitting the large plane change into the two burns can potentially result in a lower cost. In cases where the filter favours solutions with larger burns at pericentre, it can also incur in higher costs estimation for the plane change than the optimal solution.

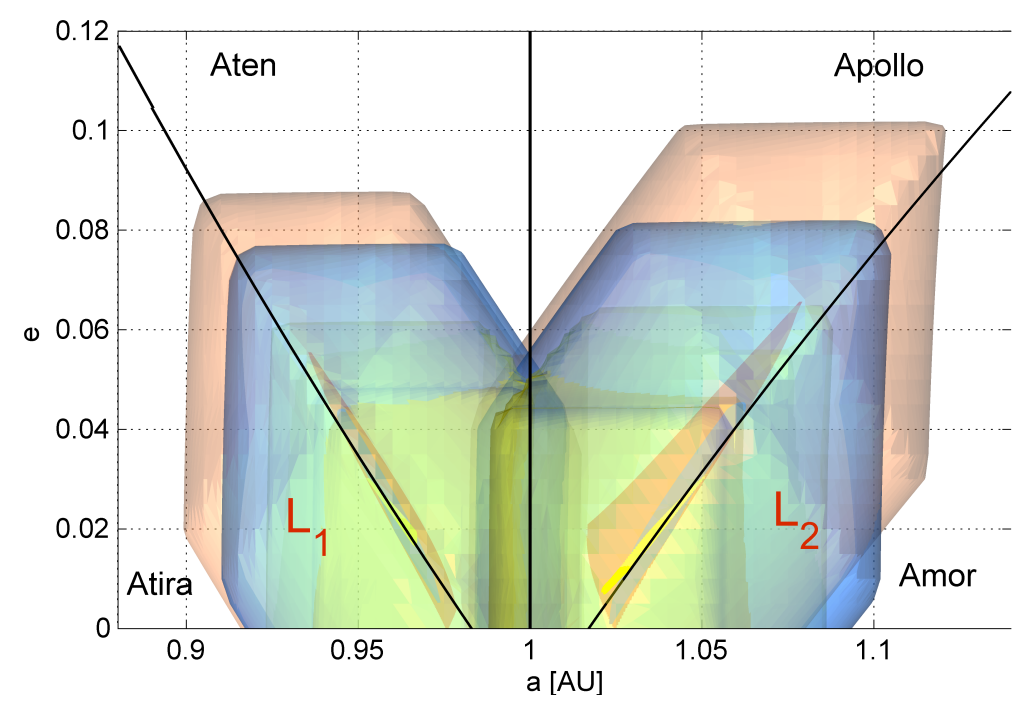

Fig. 8: Semi-major axis and eccentricity map of the capturable regions for $\mathrm{L}_{1}$ and $\mathrm{L}_{2}$. The boundaries of the main 4 families of NEO objects are also indicated. The manifold orbital elements are enclosed in the capturable regions and closely follow the Apollo-Amor and Aten-Atira divides.

\section{Capture Transfers and Mass Estimates}

For each of the filtered NEOs with estimated $\Delta v$ below $1 \mathrm{~km} / \mathrm{s}$, feasible capture transfers with arrival date in the interval 2016-2100 were obtained. The NEO orbital elements in JPL's database are only considered valid until their next close encounter with Earth.

The problem can thus be defined with 5 variables: the Lambert arc transfer time, the manifold transfer time, the insertion date at the final target orbit, the Jacobi constant of this target orbit, and a fifth discrete variable determining the point in the target orbit where the insertion takes 
place. The manifolds are discretised in terms of their Jacobi constant and their insertion point. Five hundred insertion points are considered for each LPO, which propagated backwards translate into 500 sets of orbital elements at the $\pm \pi / 8$ section.

The Lambert transfers between the asteroid initial orbit and the manifolds were optimised using EPIC, a global optimisation method that uses a stochastic search blended with an automatic solution space decomposition technique and can handle both continuous and discrete variables (see (Vasile and Locatelli, 2009) for details). Single objective optimisations with total transfer $\Delta v$ as the cost function were carried out. Trajectories obtained with EPIC were locally optimised with MATLAB's built-in function fmincon. Lambert arcs with up to 3 complete revolutions before insertion into the manifold were considered. For cases with at least one complete revolution, the two possible solutions of the Lambert problem were optimised. This implies that 7 full problem optimisations needed to be run for each NEO.

Figure 9 plots the results of the optimisation for the $L_{2}$ case together with the filter estimates. It can be observed that the filter provides in general a good approximation of the total cost to be expected. It is a useful tool to select candidates and prioritise lists of asteroids for optimisation, and to quickly predict if any newly discovered asteroid is expected to have low capture costs. Dotted lines have been added to the plot as indicators of the ideal cost of just the inclination change at a circular orbit at 1 AU. Predicted and optimised results are expected to fall above or close to these lines. The filter does however provide a quick and much more accurate estimate of the costs taking into consideration the shape of the original orbit as well as the inclination. Asteroids with capture costs smaller than 500 $\mathrm{m} / \mathrm{s}$ are indentified in the plots.

Table 2 shows the asteroids with costs lower than this selected threshold of $500 \mathrm{~m} / \mathrm{s}$. Twelve asteroids of the whole NEO catalogue can be captured at this cost, ten of them around $\mathrm{L}_{2}$ plus two Atens around $\mathrm{L}_{1}$. The table provides the orbital elements, minimum orbit intersection distance according to the JPL Small Bodies Database, and an estimate of the size of the object. This estimate is calculated with the following relation (Chesley et al., 2002):

$$
D=1329 k m \times 10^{-H / 5} p_{v}^{-1 / 2}
$$

where the absolute magnitude $H$ is provided in the JPL database, and the albedo $p_{v}$ is assumed to range from 0.05 (dark) to 0.50 (very bright icy object). 

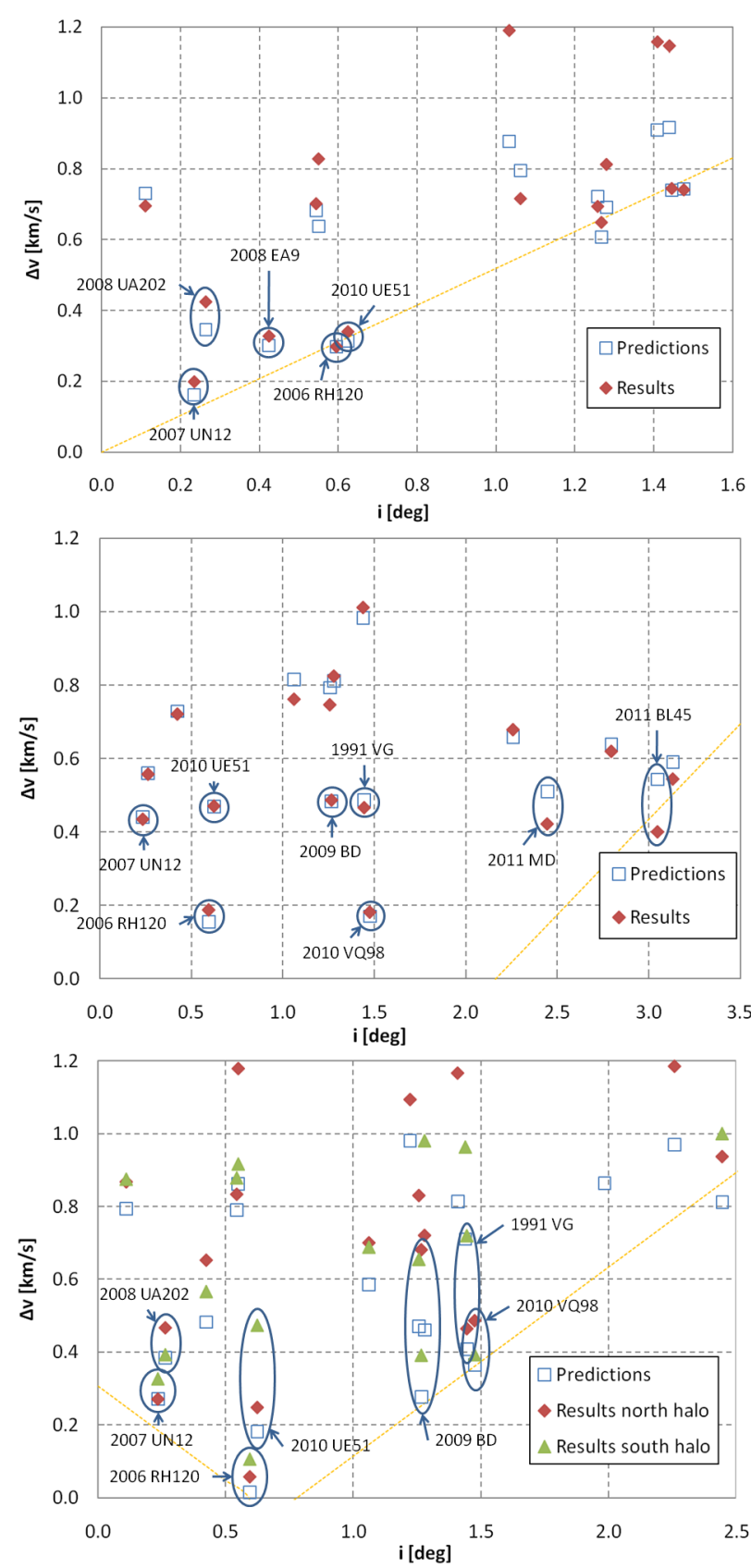

Fig. 9: Filter cost estimates and results of the optimisation for planar Lyapunov (top), vertical Lyapunov (middle) and halo orbits (bottom) around $\mathrm{L}_{2}$. Dotted lines indicate the cost of changing just the inclination. 
Table 2: NEO characteristics for transfer trajectories with $\Delta v$ below $500 \mathrm{~m} / \mathrm{s}$. The type of transfer is indicated by a 1 or 2 indicating $\mathrm{L}_{1}$ or $\mathrm{L}_{2}$ plus the letter $\mathrm{P}$ for planar Lyapunov, V for vertical Lyapunov, and $\mathrm{Hn}$ or Hs for north and south halo.

\begin{tabular}{|c|c|c|c|c|c|c|c|}
\hline & $\begin{array}{l}\mathrm{a} \\
{[\mathrm{AU}]}\end{array}$ & $\mathrm{e}$ & $\begin{array}{l}\mathrm{i} \\
\text { (Pravec } \\
\text { et al.) }\end{array}$ & $\begin{array}{l}\text { MOID } \\
\text { AU] }\end{array}$ & $\begin{array}{l}\text { Diameter } \\
{[\mathrm{m}]}\end{array}$ & Type & $\begin{array}{l}\Delta \mathrm{v} \\
{[\mathrm{m} / \mathrm{s}]}\end{array}$ \\
\hline \multirow{4}{*}{2006 RH120 } & \multirow{4}{*}{1.033} & \multirow{4}{*}{0.024} & \multirow{4}{*}{0.595} & \multirow{4}{*}{0.0171} & \multirow{4}{*}{$2.3-7.4$} & $2 \mathrm{Hs}$ & 58 \\
\hline & & & & & & $2 \mathrm{Hn}$ & 107 \\
\hline & & & & & & $2 \mathrm{~V}$ & 187 \\
\hline & & & & & & $2 \mathrm{P}$ & 298 \\
\hline \multirow{3}{*}{2010 VQ98 } & \multirow{3}{*}{1.023} & \multirow{3}{*}{0.027} & \multirow{3}{*}{1.476} & \multirow{3}{*}{0.0048} & \multirow{3}{*}{$4.3-13.6$} & $2 \mathrm{~V}$ & 181 \\
\hline & & & & & & $2 \mathrm{Hn}$ & 393 \\
\hline & & & & & & $2 \mathrm{Hs}$ & 487 \\
\hline \multirow{4}{*}{2007 UN12 } & \multirow{4}{*}{1.054} & \multirow{4}{*}{0.060} & \multirow{4}{*}{0.235} & \multirow{4}{*}{0.0011} & \multirow{4}{*}{$3.4-10.6$} & $2 \mathrm{P}$ & 199 \\
\hline & & & & & & $2 \mathrm{Hs}$ & 271 \\
\hline & & & & & & $2 \mathrm{Hn}$ & 327 \\
\hline & & & & & & $2 \mathrm{~V}$ & 434 \\
\hline \multirow{4}{*}{2010 UE51 } & \multirow{4}{*}{1.055} & \multirow{4}{*}{0.060} & \multirow{4}{*}{0.624} & \multirow{4}{*}{0.0084} & \multirow{4}{*}{$4.1-12.9$} & $2 \mathrm{Hs}$ & 249 \\
\hline & & & & & & $2 \mathrm{P}$ & 340 \\
\hline & & & & & & $2 \mathrm{~V}$ & 470 \\
\hline & & & & & & $2 \mathrm{Hn}$ & 474 \\
\hline 2008 EA9 & 1.059 & 0.080 & 0.424 & 0.0014 & $5.6-16.9$ & $2 \mathrm{P}$ & 328 \\
\hline \multirow{3}{*}{2011 UD21 } & \multirow{3}{*}{0.980} & \multirow{3}{*}{0.030} & \multirow{3}{*}{1.062} & \multirow{3}{*}{0.0043} & & $1 \mathrm{Hs}$ & 356 \\
\hline & & & & & $3.8-12.0$ & $1 \mathrm{~V}$ & 421 \\
\hline & & & & & & $1 \mathrm{Hn}$ & 436 \\
\hline 2009 BD & 1062 & 0052 & 1267 & 00053 & (4)-134 & $2 \mathrm{Hn}$ & 392 \\
\hline 2009 БD & 1.002 & 0.052 & 1.201 & 0.0053 & $4.2-15.4$ & $2 \mathrm{~V}$ & 487 \\
\hline & & & & & & $2 \mathrm{Hn}$ & 393 \\
\hline 2008 UA202 & 1.033 & 0.069 & 0.264 & $2.5 \cdot 10^{-4}$ & $2.4-7.7$ & $2 \mathrm{P}$ & 425 \\
\hline & & & & & & $2 \mathrm{Hs}$ & 467 \\
\hline 2011 BL45 & 1.033 & 0.069 & 3.049 & 0.0040 & $6.9-22.0$ & $2 \mathrm{~V}$ & 400 \\
\hline $2011 \mathrm{MD}$ & 1.056 & 0.037 & 2.446 & 0.0018 & $4.6-14.4$ & $2 \mathrm{~V}$ & 422 \\
\hline & & & & & & $1 \mathrm{P}$ & 443 \\
\hline 2000 SG344 & 0.978 & 0.067 & 0.111 & $8.3 \cdot 10^{-4}$ & $20.7-65.5$ & $1 \mathrm{Hs}$ & 449 \\
\hline & & & & & & $1 \mathrm{Hn}$ & 468 \\
\hline $1991 \mathrm{VG}$ & 1.027 & 0.049 & 1.445 & 0.0037 & $39-12.5$ & $2 \mathrm{Hs}$ & 465 \\
\hline ט 19918 & 1.021 & 0.047 & $1.4+3$ & & $3.9-12.03$ & $2 \mathrm{~V}$ & 466 \\
\hline
\end{tabular}

As expected, planar Lyapunov orbits are optimal for lower inclination NEOs, while NEOs with higher inclination favour transfers to vertical Lyapunov. Figure 10 shows an example trajectories in a co-rotating frame where the Sun-Earth line is fixed for a transfer of asteroid 2006 RH120 to a south halo orbit around $\mathrm{L}_{2}$. Close-ups of the final parts of the trajectory 
are plotted in a three-dimensional view in order to appreciate the shape of the final orbit and manifolds.

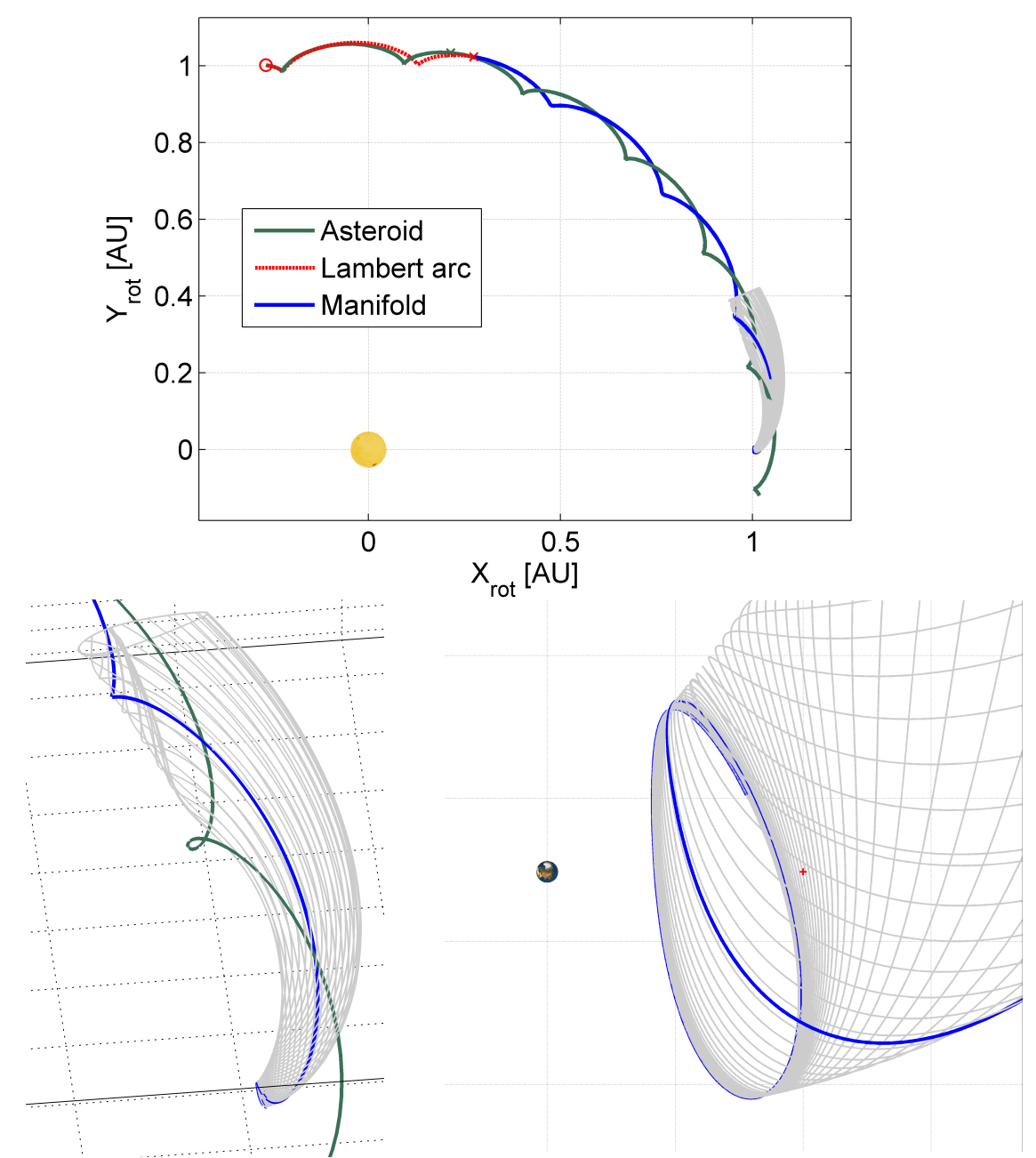

Fig. 10: Capture trajectory for asteroid 2006 RH120 to a south halo orbit. Sun and Earth are plotted 10 times their size.

Table 3 presents the best trajectory for each type of transfer for L2 and L1 (highlighted in bold in Table 2). The cheapest transfer, below $60 \mathrm{~m} / \mathrm{s}$, corresponds to a trajectory inserting asteroid 2006 RH120 into a halo orbit. Solutions to planar and vertical Lyapunov were also found for 2006 RH120 at higher costs. This agrees well with the interpretation of Fig. 6. The pruning method was also predicting that this transfer would be the 
cheapest, with a minimum estimated $\Delta v$ of $15 \mathrm{~m} / \mathrm{s}$. It is worthwhile to emphasise that the total $\Delta v$ comprises both burns at departure from the asteroid and insertion into the manifold, but it does not include any navigation costs or corrections. The NEO orbit may intersect the manifold directly, and in that case the transfer to the target orbit can be done with a single burn, as in this particular case.

The total duration of the transfers range from 3 to 7.5 years. For the longer transfers it is possible to find faster solutions with less revolutions in the Lambert arc at a small $\Delta v$ penalty.

Table 3: Capture trajectories for the lowest cost transfers to each type of LPO

\begin{tabular}{|c|c|c|c|c|c|c|c|}
\hline & & Date $[\mathrm{yy} / \mathrm{n}$ & $\mathrm{am} / \mathrm{dd}]$ & & Jacobi & Total & $\Delta \mathrm{v}[\mathrm{m} / \mathrm{s}]$ \\
\hline & & $\begin{array}{l}\text { Asteroid } \\
\text { departure }\end{array}$ & $\begin{array}{l}\text { Manifold } \\
\text { insertion }\end{array}$ & $\begin{array}{l}\mathrm{L}_{\mathrm{i}} \\
\text { arrival }\end{array}$ & $\begin{array}{l}\text { constant } \\
\text { manifold }\end{array}$ & $\begin{array}{l}\text { Durat. } \\
\text { [yr] }\end{array}$ & Dep Ins \\
\hline 2006 RH120 & $2 \mathrm{Hs}$ & $21 / 02 / 01$ & $21 / 02 / 01$ & $28 / 08 / 05$ & 3.000421 & 7.51 & 58 \\
\hline 2006 RH120 & $2 \mathrm{Hn}$ & $23 / 05 / 11$ & $24 / 02 / 20$ & $28 / 08 / 31$ & 3.000548 & 5.31 & $52 \quad 55$ \\
\hline 2010 VQ98 & $2 \mathrm{~V}$ & $35 / 02 / 14$ & $35 / 09 / 01$ & $39 / 11 / 15$ & 3.000016 & 4.75 & 177 \\
\hline 2007 UN12 & $2 \mathrm{P}$ & $13 / 10 / 22$ & $13 / 10 / 22$ & $21 / 02 / 19$ & 3.000069 & 7.33 & 199 \\
\hline 2011 UD21 & $1 \mathrm{Hs}$ & $37 / 11 / 20$ & $38 / 07 / 03$ & $42 / 07 / 19$ & 3.000411 & 4.66 & 149207 \\
\hline 2011 UD21 & $1 \mathrm{~V}$ & $36 / 07 / 20$ & $38 / 11 / 16$ & $41 / 06 / 21$ & 3.000667 & 4.92 & 226196 \\
\hline 2011 UD21 & $1 \mathrm{Hn}$ & $39 / 10 / 24$ & $40 / 06 / 15$ & $43 / 08 / 30$ & 3.000504 & 3.85 & 210226 \\
\hline 2000 SG344 & $1 \mathrm{P}$ & $24 / 02 / 11$ & $25 / 03 / 11$ & $27 / 06 / 18$ & 3.000357 & 3.35 & 195248 \\
\hline
\end{tabular}

\section{Retrieved mass estimates}

The results presented in the previous section could be used to calculate a limit in the mass that can be captured with current space technology. In order to obtain a first estimate of the size of the asteroid that could be retrieved, we can consider a basic system mass budget exercise. Assuming a spacecraft of $5500 \mathrm{~kg}$ dry mass and $8100 \mathrm{~kg}$ of propellant at the NEO (as proposed in the Keck study report for asteroid retrieval (Brophy et al., 2012)), it is possible to estimate the total asteroid mass that can be transferred. A full system budget would require a larger fuel mass to deliver the spacecraft to the target, and thus an analysis of the outbound leg, but that is beyond the scope of this work.

Results are presented for each trajectory on Table 4 for two different propulsion systems. The total mass for a high thrust engine of specific impulse 300s ranges from 44 to about 400 tons, which represents 3 to 30 times the wet mass of the spacecraft at arrival to the NEO. The trajectories presented assume impulsive burns, so in principle they are not suitable for 
low-thrust transfers. However, due to their low $\Delta v$ and long time of flight, transformation of these trajectories to low-thrust is in principle feasible, and will be considered in future work. If a similar cost trajectory could be flown with a low-thrust engine of higher specific impulse (3000s) the asteroid retrieved mass would be over ten times that of the high-thrust case, up to an impressive 4000 tons in the case of a hypothetical transfer from the orbit of 2006 RH120 to a halo orbit. That is beyond the maximum estimated size of this particular asteroid. Even if losses of $600 \%$ were assumed in the transformation from high to low thrust (and this could well be the case given the mass of the object intended for transfer) the estimated diameter would still be greater than the $7.4 \mathrm{~m}$ maximum expected size of asteroid 2006 RH120.

For the average NEO density and assuming spherical bodies, the equivalent diameter of the asteroid that can be captured is also included in the table. This shows that reasonably sized boulders of 3-7 m diameter, or small asteroids of that size, could be captured with this method. The capture of entire bodies of larger size is still challenging, but the derived size of a few of the candidates fall actually within this range. With the higher specific impulse, the only NEO in the table that fails to meet the capturable range shown in Table 4 is 2000 SG344, with a derived size in the range of 20 to 65 meters.

Table 4: Retrieved mass estimates with current space technology.

\begin{tabular}{|c|c|c|c|c|c|c|}
\hline & & \multirow{2}{*}{$\begin{array}{l}\text { Total } \\
\Delta \mathrm{v} \\
{[\mathrm{m} / \mathrm{s}]}\end{array}$} & \multicolumn{2}{|l|}{ Isp $=300 \mathrm{~s}$} & \multicolumn{2}{|l|}{$\mathrm{Isp}=3000 \mathrm{~s}$} \\
\hline & & & $\begin{array}{l}\text { Mass } \\
{[\mathrm{kg}]}\end{array}$ & $\begin{array}{l}\varnothing \\
{[\mathrm{m}]}\end{array}$ & $\begin{array}{l}\text { Mass } \\
{[\mathrm{kg}]}\end{array}$ & $\begin{array}{l}\varnothing \\
{[\mathrm{m}]}\end{array}$ \\
\hline 2006 RH120 & $2 \mathrm{Hs}$ & 58 & 398144 & 6.64 & 4067256 & 614.40 \\
\hline 2006 RH120 & $2 \mathrm{Hn}$ & 107 & 213657 & 7.39 & 2222273 & 311.77 \\
\hline 2010 VQ98 & $2 \mathrm{~V}$ & 181 & 121879 & 4.47 & 1304330 & 9.86 \\
\hline 2007 UN12 & $2 \mathrm{P}$ & 199 & 110313 & 4.33 & 1188630 & 9.56 \\
\hline 2011 UD21 & $1 \mathrm{Hs}$ & 356 & 57441 & 3.48 & 659549 & 97.85 \\
\hline 2011 UD21 & $1 \mathrm{~V}$ & 422 & 47017 & 3.26 & 555160 & 7.42 \\
\hline 2011 UD21 & $1 \mathrm{Hn}$ & 436 & 45236 & $5 \quad 3.21$ & 537325 & $5 \quad 7.34$ \\
\hline 2000 SG344 & $1 \mathrm{P}$ & 443 & 44380 & 3.19 & 528741 & 17.30 \\
\hline
\end{tabular}

\section{Overview of the Selected Candidates}

The capture candidates are all of small size (perhaps with the exception of 2000 SG344), which is ideal for a technology demonstrator retrieval 
mission. In fact, seven of them fit the small-Earth approachers (SEA) definition by Brasser and Wiegert (2008). They showed, focusing on object $1991 \mathrm{VG}$, that the orbit evolution of these type of objects is dominated by close encounters with Earth, with a chaotic variation in the semi-major axis over long periods of time. A direct consequence of this is that reliable capture transfers can only be designed with accuracy over one synodic period, before the next encounter with Earth changes the orbital elements significantly. One could argue that finely tuning these encounters could also be used to shepherd these objects into trajectories that have a lower cost to be inserted into a manifold (Sanchez and McInnes, 2011b).

The candidates NEOs in Table 2 are well-known, and there has been speculation about the origin of a few of them, including the possibility that they were man-made objects (spent upper stages), lunar ejecta after an impact (Tancredi, 1997, Chodas and Chesley, 2001, Brasser and Wiegert, 2008, Kwiatkowski et al., 2009), or even an alien probe (Steel, 1995). In particular object 2006 RH120 has been thoroughtly studied (Kwiatkowski et al., 2009, Granvik et al., 2011), as it was a temporarily captured orbiter that was considered the "second moon of the Earth" until it finally escaped the Earth in July 2007. Granvik shows that the orbital elements of 2006 RH120 changed from being an asteroid of the Atens family pre-capture, to an Apollo post-capture, having followed what we refer to in previous sections as a transit orbit inside Earth's Hill sphere. An additional object in the list, $2007 \mathrm{UN} 12$, is also pointed out by Granvik as a possible candidate to become a TCO (Temporarily Captured Orbiter).

Regarding their accessibility, a recent series of papers (Adamo et al., 2010, Barbee et al., 2010, Hopkins et al., 2010) considered up to 7 of the above objects as possible destinations for the first manned mission to a NEO (and the other 5 were not discovered at the time). They proposed human missions during the same close approaches as the capture opportunities calculated. However, the arrival dates at the asteroids are later than the required departure date for the capture, so their outbound legs could not apply to our proposed capture trajectories. An additional study by Landau and Strange (2011) presents crewed mission trajectories to over 50 asteroids. It shows that a mission to 6 of the considered asteroids is possible with a low-thrust $\Delta v$ budget between 1.7 and $4.3 \mathrm{~km} / \mathrm{s}$. The costs presented are for a return mission of a spacecraft with a dry mass of 36 tons (including habitat) in less than 270 days. A longer robotic mission with a final mass at the NEO of $13,600 \mathrm{~kg}$ and a manifold capture as the one proposed would result in much lower fuel costs as the thrust-tomass ratio increases. NASA also publishes the Near-Earth Object Human Space Flight Accessible Target Study (NHATS) list (Abell et al., 2012), which will be continuously updated and identifies potential candidate 
objects for human missions to asteroids. The NEOs are ranked according to the number of feasible return trajectories to that object found by an automated search within certain constaints. Eleven of our 12 capturable objects appear in the top 25 of NASA's NHATS list as of September 2012, seven of them in the top 10. This seems to indicate that the objects found by our pruning and optimisation are indeed easily accessible, even if the outbound part of the trajectory was not considered in our calculations.

\section{Method Limitations}

One of the first objections that can be raised to the approach presented involves some of the simplifications in the model. The main simplifying assumptions are placing the Earth in a circular orbit, assuming Keplerian propagation for the NEOs orbital elements until the next close encounter with Earth, and not including other types of perturbations, in particular the Moon third body perturbation. While the influence of the first two assumptions should be relatively small, and the trajectories obtained can be used as first guesses for a local optimisation with a more complex model with full Earth and NEOs ephemerides, not including the Moon as a perturbing body can have a much greater influence. Granvik (2011) shows that the Moon plays an important role in the capture of TCO, and so the trajectories of the manifolds would be also affected by it. The lunar third body perturbation can also strongly influence the stability of LPOs, in particular large planar Lyapunov orbits, and it could render some of them unsuitable for target orbits. However, the general behaviour and the type of NEOs that can be captured are not expected to change. Other perturbations, such as the changes in the orbit of small bodies affected by solar radiation pressure are of little importance within the timescales considered.

Other capture possibilities, e.g. by means of a single or double lunar swingby, have not been studied and are outside of the scope of this chapter, but they may provide even cheaper asteroid retrieval opportunities.

\section{Conclusions}

The possibility of capturing a small NEO or a segment from a larger object would be of great scientific and technological interest in the coming decades. It is a logical stepping stone towards more ambitious scenarios of asteroid exploration and exploitation, and possibly the easiest feasible attempt for humans to modify the Solar System environment outside of Earth, or attempting any large scale macro-engineering project. 
This analysis has shown that the retrieval of a full asteroid is well within today's technological capabilities, and that there exists a series of objects that can be easily captured into libration point orbits. Taking advantage of this, the utilisation of asteroid resources may be a viable means of providing substantial mass in Earth orbit for future space ventures. Despite the largely incomplete survey of very small objects, the current known population of asteroids provides a good starting platform to begin with the search for easily capturable objects. With this goal, a robust methodology for systematic pruning of a NEO database and optimisation of capture trajectories through the hyperbolic invariant stable manifold into different types of LPO around $\mathrm{L}_{1}$ and $\mathrm{L}_{2}$ has been implemented and tested. Twelve possible candidates for affordable full asteroid retrieval missions have been identified among known NEOs with capture opportunities during the next 30 years with a cost below $500 \mathrm{~m} / \mathrm{s}$. Transfers to the libration points region have been calculated for all these targets. These transfers enable the capture of bodies within 3-7 meters diameter with low propellant costs.

The proposed method can be easily automated to prune the NEO database on a regular basis, as the number of objects in orbits of interest is expected to grow asymptotically with the new efforts in asteroid detection. Any new occurrence of a low-cost candidate asteroid can be optimised to obtain the next available phasing and transfer opportunities and the optimal target LPO.

Moreover Sun-Earth LPOs can also be considered as natural gateways to the Earth system. Thus, the problem to transfer an asteroid to an Earth or Moon centred orbit can be decoupled into the initial phase of inserting the asteroid into a stable invariant manifold and then providing the very small manoeuvres required to continue the transit into the Earth system. While a method to find optimal Sun-Earth LPO capture trajectories and possible targets has been defined, the transit trajectories can potentially allow the asteroid to move to the Earth-Moon $\mathrm{L}_{1} / \mathrm{L}_{2}$ or other locations within cis-lunar space taking advantage of heteroclinic connections between collinear points.

This analysis has also shown the costs of accessing the capture material at the Sun-Earth collinear equilibrium points. Given the costs associated with reaching the Sun-Earth LPOs, one can imagine the scientific and exploitation advantages of bringing asteroids close to Earth, as oppose to reaching them on their unperturbed heliocentric orbits. 


\section{Acknowledgements}

We would like to thank Elisa Maria Alessi for her valuable comments and inputs to this work. We would also like to acknowledge the use of the Faculty of Engineering High Performance Computer Facility, University of Strathclyde. The work reported was supported by European Research Council grant 227571 (VISIONSPACE).

\section{References}

Abell PA, Barbee BW, Mink RG, Adamo DR, Alberding CM, Mazanek DD et al. (2012) The Near-Earth Object Human Space Flight Accessible Targets Study (NHATS) List of Near-Earth Asteroids: Identifying Potential Targets For Future Exploration, NASA,

Adamo DR, Giorgini JD, Abell PA, and Landis RR (2010) Asteroid Destinations Accessible for Human Exploration: A Preliminary Survey in Mid-2009. Journal of Spacecraft and Rockets 47, 994-1002.

Alessi EM (2010) The Role and Usage of Libration Point Orbits in the Earth Moon System [PhD Thesis], Universitat de Barcelona, Barcelona.

Aravind PK (2007) The physics of the space elevator. American Journal of Physics 75,

Baoyin H-X, Chen Y, and Li J-F (2010) Capturing near earth objects. Research in Astronomy and Astrophysics 10, 587-98.

Barbee BW, Espositoy T, III EP, Hur-Diaz S, Mink RG, and Adamo DR (2010) A Comprehensive Ongoing Survey of the Near-Earth Asteroid Population for Human Mission Accessibility. In AIAA Guidance, Navigation, and Control Conference, Toronto, Ontario, Canada.

Bewick R, Sanchez JP, and McInnes CR (2012) The feasibility of using an L1 positioned dust cloud as a method of space-based geoengineering. Advances in Space Research 49, 1212-28.

Bottke WF, Morbidelli A, Jedicke R, Petit J-M, Levison HF, Michel P et al. (2002) Debiased Orbital and Absolute Magnitude Distribution of the NearEarth Objects. Icarus 156, 399-433.

Brasser R and Wiegert P (2008) Asteroids on Earth-like orbits and their origin. Monthly Notices of the Royal Astronomical Society 386, 2031-8.

Brophy J, Culick F, Friedman L, Allen C, Baughman D, Bellerose J et al. (2012) Asteroid Retrieval Feasibility Study, Keck Institute for Space Studies, Califonia Institute of Technology, Jet Propulsion Laboratory, Pasadena, California.

Ceriotti M and McInnes C (2012) Natural and sail-displaced doubly-symmetric Lagrange point orbits for polar coverage. Celestial Mechanics and Dynamical Astronomy 1-30. 
Chesley SR, Chodas PW, Milani A, Valsecchi GB, and Yeomans DK (2002) Quantifying the Risk Posed by Potential Earth Impacts. Icarus 159, 423-32.

Chodas PW and Chesley SR (2001) 2000 SG344: The Story of a Potential Earth Impactor. Bulletin of the American Astronomical Society 33, 1196.

Edward TL and Stanley GL (2005) Gravitational Tractor for Towing Asteroids. Nature 438, 177-8.

Farquhar RW (1967) Station-keeping in the vicinity of collinear libration points with an application to a Lunar communications problem. In Space Flight Mechanics. Vol. 11, pp. 519-35. American Astronautical Society, New York.

Gómez G, Jorba A, Simó C, and Masdemont J (2001) In Dynamics and Mission Design Near Libration Points: Advanced Methods for Collinear Points. Vol. 3, World Scientific Publishing, Singapore.

Gómez G, Llibre J, Martínez R, and Simó C (2000) In Dynamics and Mission Design Near Libration Point Orbits-Fundamentals: The Case of Collinear Libration Points. Vol. 1, World Scientific Publishing, Singapore.

Gómez G, Marcote M, and Mondelo JM (2005) The invariant manifold structure of the spatial Hill's problem. Dynamical Systems 20, 115-47.

Granvik M, Vaubaillon J, and Jedicke R (2011) The population of natural Earth satellites. Icarus 218, 262-77.

Hasnain Z, Lamb C, and Ross SD (2012) Capturing near-Earth asteroids around Earth. Acta Astronautica 81, 523-31.

Hénon M (1973) Vertical Stability of Periodic Orbits in the Restricted Problem, I. Equal Masses. Astronomy and Astrophysics 28, 415-26.

Hopkins J, Dissel A, Jones M, Russell J, and Gaza R (2010) Plymouth Rock: An Early Human Mission to Near Earth Asteroids Using Orion Spacecraft, Lockheed Martin Corporation,

Howell KC (2001) Families of Orbits in the Vicinity of Collinear Libration Points. Journal of the Astronautical Sciences 49, 107-25.

Howell KC and Pernicka HJ (1993) Stationkeeping Methods for Libration Point Trajectories. Journal of Guidance Control Dynamics 16, 151-9.

Kawaguchi J, Fujiwara A, and Uesugi T (2008) Hayabusa-Its technology and science accomplishment summary and Hayabusa-2. Acta Astronautica 62, 639-47.

Kleiman LA (1968) In Project Icarus: an MIT Student Project in Systems Engineering, The MIT Press, Cambridge, Massachusetts.

Koon WS, Lo MW, Marsden JE, and Ross SD (2008) In Dynamical systems, the three-body problem and space mission design. Vol., Marsden Books,

Koon WS, Lo MW, Marsden JE, and Ross SD (2000) Heteroclinic Connections Between Periodic Orbits and Resonance Transitions in Celestial Mechanics. Chaos 10, 427-69.

Kwiatkowski T, Kryszczynska A, Polinska M, Buckley DAH, O'Donoghue D, Charles PA et al. (2009) Photometry of 2006 RH120: an asteroid temporary captured into a geocentric orbit. Astronomy and Astrophysics 495, 967-74. 
Landau D and Strange N (2011) Near-Earth Asteroids Accesible to Human Exploration with High-Power Electric Propulsion. In AAS/AIAA Astrodynamics Specialist Conference, Girdwood, Alaska.

Lewis JS (1996) In Mining the sky: untold riches from asteroids, comets and planets, Helix Books/Perseus Books Reading, Massachusetts.

Mainzer A, Grav T, Bauer J, Masiero J, McMillan RS, Cutri RM et al. (2011) NEOWISE Observations of Near-Earth Objects: Preliminary Results. The Astrophysical Journal 743,

Massonnet D and Meyssignac B (2006) A captured asteroid : Our David's stone for shielding earth and providing the cheapest extraterrestrial material. Acta Astronautica 59, 77-83.

Pearson J, Oldson J, and Levin E (2006) Earth rings for planetary environment control. Acta Astronautica 58, 44-57.

Pravec P, Scheirich P, Kušnirák P, Šarounová L, Mottola S, Hahn G et al. (2006) Photometric survey of binary near-Earth asteroids. Icarus 181, 63-93.

Richardson DL (1980) Halo orbit formulation for the ISEE-3 mission. Journal of Guidance Control Dynamics 3,

Sanchez JP and Colombo C (2012) Impact Hazard Protection Efficiency by a Small Kinetic Impactor Journal of Spacecraft and Rockets In Press,

Sanchez JP, García-Yárnoz D, and McInnes CR (2012) Near-Earth Asteroid Resource Accessibility and Future Capture Missions Opportunities. In Global Space Exploration Conference 2012, International Austranautical Federation, Washington DC, USA.

Sanchez JP and McInnes CR (2011a) Asteroid Resource Map for Near-Earth Space. Journal of Spacecraft and Rockets 48, 153-65.

Sanchez JP and McInnes CR (2012) An asteroid resource map: Assessing future opportunities,

Sanchez JP and McInnes CR (2011b) On the Ballistic Capture of Asteroids for Resource Utilization. In 62nd International Astronautical Congress, IAF, Cape Town, SA.

Scheeres DJ and Schweickart RL (2004) The Mechanics of Moving Asteroids. In Planetary Defense Conference. AIAA 2004-1446, American Institute of Aeronautics and Astronautics, Orange County, California.

Shapiro II, A'Hearn M, Vilas F, and et.al. (2010) Defending Planet Earth: NearEarth Object Surveys and Hazard Mitigation Strategies, National Research Council, Washington, D.C.

Simó C, Gómez G, Llibre J, Martínez R, and Rodríguez J (1987) On the optimal station keeping control of halo orbits. Acta Astronautica 15, 391-7.

Steel D (1995) SETA and 1991 VG. The Observatory 115, 78-83.

Struck C (2007) The feasibility of shading the greenhouse with dust clouds at the stable lunar Lagrange points. Journal of the British Interplanetary Society (JBIS) 60, 82-9.

Szebehely V (1967) In Theory of orbits, Academic Press, New York.

Tancredi (1997) An asteroid in a Earth-like orbit. Celestial Mechanics and Dynamical Astronomy 69, 119-32 
Tsiolkovsky KE (1903) The Exploration of Cosmic Space by Means of Reaction Devices. Scientific Review

Vasile $M$ and Locatelli M (2009) A hybrid multiagent approach for global trajectory optimization. Journal of Global Optimization 44, 461-79.

Veres P, Jedicke R, Wainscoat R, Granvik M, Chesley S, Abe S et al. (2009) Detection of Earth-impacting asteroids with the next generation all-sky surveys. Icarus 203, 472-85.

Zagouras C and Markellos VV (1977) Axisymmetric Periodic Orbits of Restricted Problem in Three Dimensions. Astronomy and Astrophysics 59, 79-89. 